\title{
Probing post-AGB metamorphosis with NIR Adaptive Optics Imaging
}

\author{
C. Sánchez Contreras ${ }^{1}$, D. Le Mignant ${ }^{2}$, R. Sahai ${ }^{3}$, \\ F. H. Chaffee ${ }^{2}$, and M. Morris ${ }^{4}$ \\ ${ }^{1}$ Dpto. de Astrofísica Molecular e Infrarroja, IEM-CSIC, \\ Serrano 121, 28006 Madrid, Spain \\ email: carmen@damir.iem.csic.es \\ ${ }^{2}$ W. M. Keck Observatory, \\ 65-1120 Mamalahoa Highway, Kamuela, HI 96743, USA \\ ${ }^{3}$ Jet Propulsion Laboratory/California Institute of Technology, MS 183-900, \\ Pasadena, CA 91109, USA \\ ${ }^{4}$ Department of Physics \& Astronomy, UCLA, \\ Box 951562, Los Angeles, CA 90095-1547, USA
}

\begin{abstract}
We present 1.6-4.7 $\mu \mathrm{m}$ images of PPNe and young PNe recently obtained with the Keck Adaptive Optics (AO) system. These observations provide higher angular resolution and probe deeper into the dusty envelopes of post-AGB objects than HST images and, therefore, show with unprecedented detail the morphology of these nebulae. Some objects have limbbrightened lobes displaying a remarkable point-like symmetry, which suggests the presence of underlying precessing jets that may be carving out the nebular lobes. Our images also show a very rich structure at the very small scale of $\sim 0$. 1 , including jet-like features, arcs, searchlight beams, as well as faint, extended halos in a number of objects.
\end{abstract}

Keywords. instrumentation: adaptive optics, stars: AGB and post-AGB, stars: imaging, stars: mass loss, planetary nebulae: general, infrared: stars

\section{Introduction}

The shaping of planetary nebulae $(\mathrm{PNe})$ is probably the most exciting yet least understood problem in the late evolution of $1-8 M_{\odot}$ stars. In recent years, $\sim 0.0^{\prime \prime} 1$-resolution imaging with HST has provided many new insights into the evolution from the AGB to the PN phase through the intermediate evolutionary stage of pre-planetary nebula (PPN). In light of the many structural details revealed by HST data, e.g., the multiple lobes and the high degree of point symmetry of jets observed in most young $\mathrm{PNe}$, and PPNe, Sahai \& Trauger (1998) hypothesized that fast collimated outflows are the primary agent for setting the stage in the shaping of PNe, rather than a pre-existing equatorial density enhancement in the AGB CEs, as assumed in the popular Generalized Interacting Stellar Winds (GISW) model (Balick 1987).

Since most PPNe are very small in angular extent $\left(\lesssim 5^{\prime \prime}\right)$ with rich structure at a scale $\lesssim 0$. 1 , and because the shaping/collimation mechanism operates very close to the central star, $\lesssim 10^{16} \mathrm{~cm}$ (Balick \& Frank 2002; Sánchez Contreras \& Sahai 2001), high-angular resolution observations are required to make progress in our understanding of the massloss processes mediating the AGB-to-PN transition. Also, observing at long wavelengths is desirable, since the dusty envelopes of most post-AGB objects are very optically thick. 


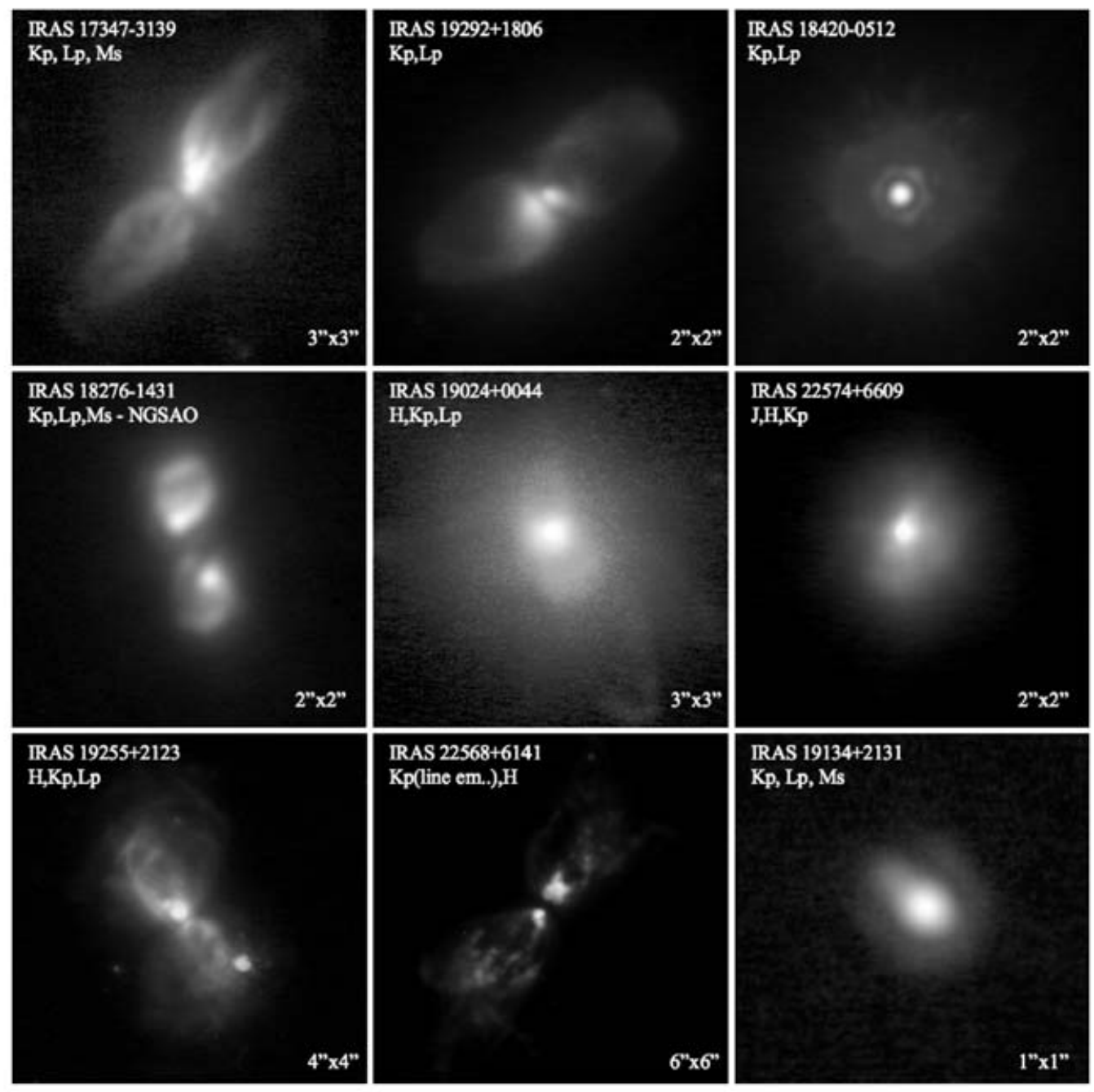

Figure 1. False-color AO images of selected nebulae in different NIR bands obtained with Keck. All images (except for IRAS 18276) were obtained in LGS mode. [Color figure available online.]

\section{Observations and Results}

We have imaged several post-AGB nebulae through the filters $J(1.25 \mu \mathrm{m}), H(1.63 \mu \mathrm{m})$, $K_{\mathrm{p}}(2.12 \mu \mathrm{m}), L_{\mathrm{p}}(3.8 \mu \mathrm{m})$, and $M_{\mathrm{s}}(4.7 \mu \mathrm{m})$ with the W. M. Keck II 10-m telescope using the NIRC2 Camera with AO in its Laser Guide Star (LGS) and Natural Guide Star (NGS) modes (Fig. 1; see also Le Mignant et al., this conference). Observations were performed in different runs from January through August 2005.

All PPNe and PNe in our sample display bipolar or multipolar morphologies. The limbbrightened appearance of the lobes in several objects suggests that these are bubble-like structures with dense walls and tenuous interiors, presumably excavated by jet-like winds. Point-symmetric lobes/features are found in some objects, which suggests the presence of underlying precessing jets. Our images also reveal significant structure inside the lobes at the very small scale of $\sim 0$.. 1 , arc-like features, searchlight beams, as well as faint, extended halos in a number of objects. The $K_{\mathrm{p}}$ image of the young PN IRAS 22568 is 
strongly contaminated by line emission $\left[\mathrm{H}_{2} 1-0 \mathrm{~S}(1)\right.$ ?], which probably traces the shocks responsible for the nebular shaping.

\subsection{IRAS $18276-1431$}
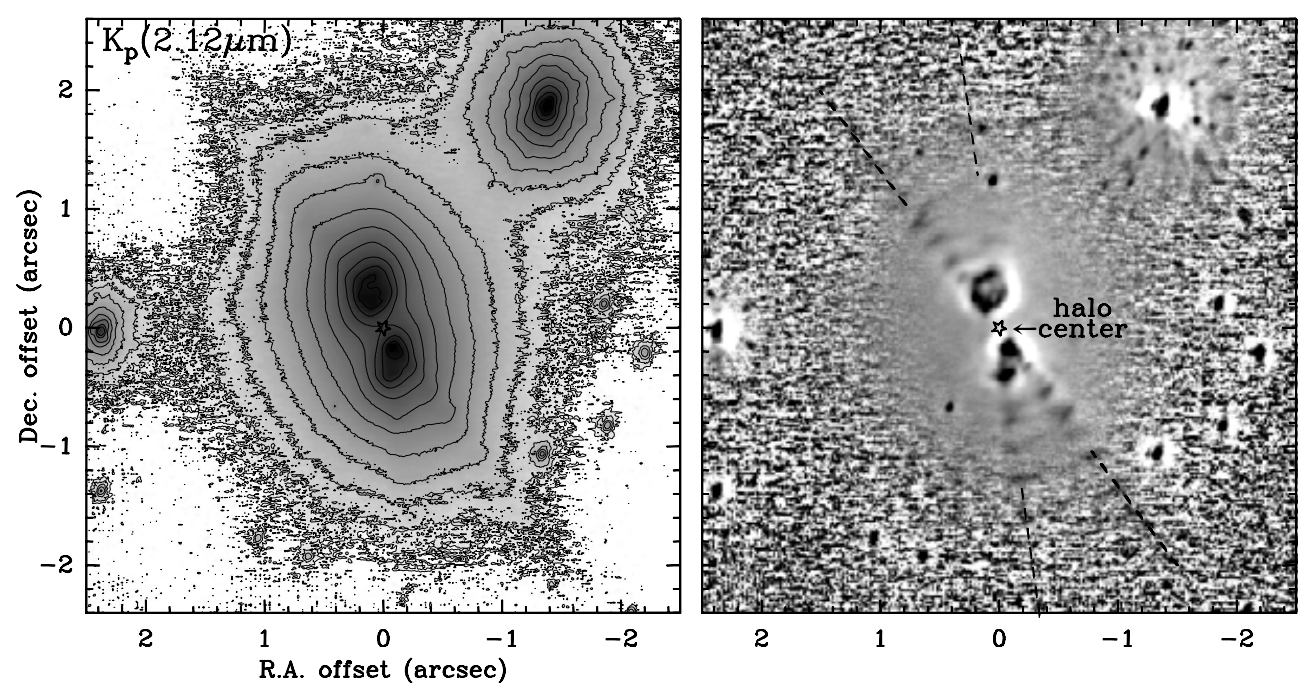

Figure 2. Left) $\mathrm{K}_{\mathrm{p}}$ image of IRAS 18276. Right) A sharpened version of the $\mathrm{K}_{\mathrm{p}}$ image to show the "searchlight beams" (indicated by dashed lines) and "arcs".

IRAS 18276-1431 (OH 17.7-2.0) is an OH/IR star rapidly evolving to the PN phase. It displays a clear bipolar morphology in our AO images (Figs. 1 \& 2 and Sánchez Contreras et al., in preparation), with two lobes oriented along $\mathrm{PA} \sim 20^{\circ}$ separated by a dark waist and surrounded by a faint $4.5 \times 3$." 4 halo. The surface brightness profile in the halo along its major axis follows a power-law $\propto r^{-4}$ ( $r$ being the radial distance to the center), indicating a density power law $\propto r^{-3}$ and, consequently, an increasing mass-loss rate with time. Our deep $K_{\mathrm{p}}$ image reveals two pairs of radial "searchlight beams" emerging from the nebula center, similar to those in CRL 2688 (Sahai et al. 1998). We also find several arc-like features with a mean separation of $\sim 0^{\prime \prime} .22$ and a brightness contrast of $\sim 10 \%$; some of the arcs appear to intersect each other. A preliminary analysis of the NIR colors suggests the presence of $\sim 500 \mathrm{~K}$ dust illuminating the nebula.

\subsection{IRAS 22574+6609}

IRAS 22574+6609 is a C-rich PPN with a likely F-G central star and weak $\mathrm{H}_{2}$ 1-0 $S(1)$ emission (e.g. Kelly \& Hrivnak 2005). Our AO images (Figs. 1\&3) show a rather irregular nebula formed by a series of blobs, which have their counterpart in the HST F814W image ( $\mathrm{Su}$ et al. 2001), surrounded by a faint halo. The brightness contrast between the intensity peak $(\mathrm{P})$ and the halo increases with wavelength; the opposite behavior is observed in the other blobs, which are not seen in the $K_{\mathrm{p}}$ image. The location of $\mathrm{P}$ is the same in all our images and does not coincide with the center of the halo, which is expected to pinpoint the illuminating source (Fig. 3). The center of the halo lies at different positions in the $J, H$, and $K_{\mathrm{p}}$ images. This may indicate that there are two sources illuminating the nebula, however, more observations (e.g. imaging polarimetry) are needed to test this scenario. Our color maps show that the north-east side of the nebula is redder than the south-west one, which suggests that the symmetry axis of the nebula may run along $\mathrm{PA} \approx 30^{\circ}$. 

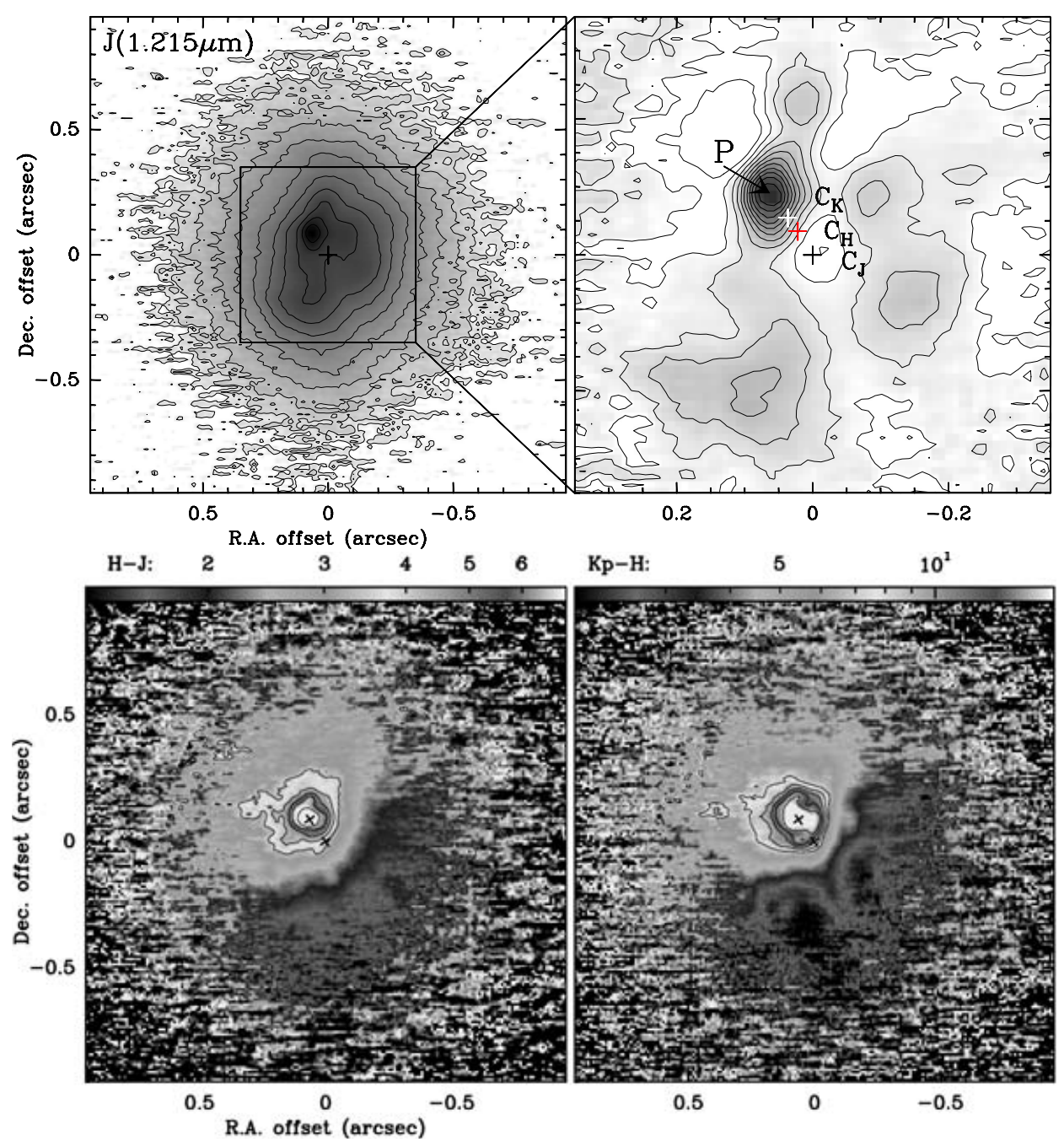

Figure 3. Top-Left) $J$ image of IRAS 22574. Top-Right) Sharpened $J$ image to show the inner structure. The center of the halo in the $J, H$, and $K_{\mathrm{p}}$ images $\left(\mathrm{C}_{\mathrm{J}}, \mathrm{C}_{\mathrm{H}}\right.$, and $\left.\mathrm{C}_{\mathrm{K}}\right)$ is indicated by crosses whose size represent the errors in the position. Bottom) $H-J$ (left) and $K_{\mathrm{p}}-H$ (right) color maps. The crosses indicate the position of $\mathrm{P}$ and $\mathrm{C}_{\mathrm{J}}$. [Color figure available online.]

\section{Acknowledgements}

CSC is supported by the Spanish MCyT under project DGES/AYA2003-02785. RS is supported by NASA under STScI grants GO-09463.01-A and GO-09801.01-A.

\section{References}

Balick, B. 1987, AJ 94, 671

Balick, B. \& Frank, A. 2002, A\&AR 40, 439

Kelly, D. M. \& Hrivnak, B. J. 2005, ApJ 629, 1040

Sahai, R. \& Trauger, J.T. 1998, AJ 116, 1357

Sahai, R., Hines, D. C., Kastner, J. H., et al. 1998, ApJ 492, L163

Sánchez Contreras, C. \& Sahai, R. 2001, ApJ 553, L173

$\mathrm{Su}$, K. Y. L., Hrivnak, B. J. \& Kwok, S. 2001, AJ 122, 1525 